\title{
Primary infertility in nuclear industry employees: report from the nuclear industry family study
}

\author{
P Doyle, E Roman , N Maconochie, G Davies, P G Smith , V Beral
}

\begin{abstract}
Objective-To determine whether men and women who are occupationally exposed to ionising radiation are at increased risk of primary infertility.

Methods-A cross sectional analysis of reproductive outcome was reported in an occupational cohort of nuclear industry workers from the Atomic Energy Authority, Atomic Weapons Establishment, and British Nuclear Fuels in the United Kingdom. Data on employment and radiation monitoring supplied by employers were linked to data obtained from self administered questionnaires sent to all current employees and a sample of past workers. A total of 5353 men and 603 women aged 40 years or more at the time of survey whose first pregnancy, or attempt at pregnancy, had occurred after first employment in the nuclear industry were recruited to the study. Primary infertility was defined as consultation with a doctor about the failure to achieve at least one viable ( 24 weeks gestational age or more) pregnancy. Results-2.6\% Of men and 3.7\% of women reported primary infertility. For men, this proportion did not differ by amount of radiation monitored or by dose received. The prevalence of infertility was higher among monitored women than nonmonitored women, but not significantly so and the numbers were too small to draw any firm conclusions.

Conclusions-No support was found for a hypothesis linking exposure to low level ionising radiation among men with primary infertility. There was weak evidence of an association in women, but the relatively few monitored women prevented detailed examination of these data. (Occup Environ Med 2001;58:535-539)
\end{abstract}

Keywords: infertility; nuclear industry

Exposure of mammalian germ cells to high doses of ionising radiation can cause interruption of spermatogenesis and the death of mature oocytes, leading to severe impairment of male and female fertility. ${ }^{1}$ Whether or not exposure to low dose radiation can influence fertility is less clear, but it is possible that exposure could result in reduced gamete viability, or the formation of a lethally damaged embryo that dies before the pregnancy is recognised. ${ }^{2}$ In human populations, such effects would result in increased levels of infertility-the inability to achieve a recognised pregnancy.
Main messages

- The aim was to determine whether men and women who are exposed to low-level ionising radiation at work are at increased risk of primary infertility. $2.6 \%$ of men and $3.7 \%$ women aged 40 or more reported primary infertility.

- For men, we found no support for a hypothesis linking exposure to low level ionising radiation with primary infertility.

- For women, the prevalence of infertility was higher among monitored women than non-monitored women, but not significantly so and the numbers were too small to draw any firm conclusions.

Few epidemiological studies have examined infertility relative to exposure to low level ionising radiation. This is partly because infertility is difficult to measure and definitions vary widely, and partly because exposure to artificial sources of ionising radiation is rare. Hence, investigations have been limited to specific groups: survivors of the atomic bombs in Japan $^{3}$; patients exposed to diagnostic or therapeutic radiation in childhood or early adulthood $^{4-7}$; and workers with occupational exposure to ionising radiation. ${ }^{89}$ The present study was conducted among workers in the nuclear industry.

The United Kingdom nuclear industry family study was set up to investigate possible links between reproductive and child health and parents' exposure to ionising radiation at work. ${ }^{10-12}$ Data were collected on reproductive histories and the health of offspring of male and female employees in the nuclear industry throughout the United Kingdom, including the British Nuclear Fuel plant at Sellafield. Results of the analysis of childhood cancer, ${ }^{11}$ fetal death, and congenital malformation ${ }^{12}$ have been reported previously. Analyses of infertility relative to parental occupational exposure to ionising radiation are presented here.

Policy implications

- These results suggest that exposure to low level ionising radiation within the nuclear industry of the United Kingdom has no detrimental effect on male fertility.

- There were insufficient data to allow firm conclusions about infertility in female nuclear industry workers. 
Participants and methods

MAIN SURVEY

Detailed information about the investigation is given elsewhere..$^{10-12}$ In summary, the study was a survey of the reproductive health of United Kingdom nuclear workers and the health of their children. Participants were current and past employees of three United Kingdom authorities: the Atomic Weapons Establishment, the Atomic Energy Authority, and British Nuclear Fuels. Between 1993 and 1996, all current employees of these three authorities, and past employees of the Atomic Energy Authority and British Nuclear Fuels who were aged under 75 years old and whose details were recorded on the company's pensions database, were sent a postal questionnaire. The questionnaire requested information about reproductive history and child health, and included questions on consulting a doctor for difficulties in achieving a pregnancy. After undelivered post was excluded, the response rate was $82 \%$ for men and $88 \%$ for women. Respondents who had never tried to have children, were asked simply to tick a box indicating this and send the questionnaire back: of those returning a questionnaire, 33\% of men and $42 \%$ of women had ticked the box and these subjects were excluded from all subsequent analyses.

Each subject was assigned a unique personal number which was used to link their survey data to industry employment and radiation monitoring records (including annual radiation dose). All employees who are potentially at risk of exposure to external ionising radiation are required to wear personal dosimeters (film badges or thermoluminescent devices) to record the amount of exposure, and for regulatory and managerial purposes nuclear establishments maintain records of all such monitoring. Workers who had at least one such radiation record are referred to in this study as "monitored workers". Natural background radiation was excluded and appropriate adjustments were made for factors such as the minimum detection dose value (threshold dose) of the dosimeter and for missing badges. ${ }^{10}$ Also, employees who are potentially exposed to radioactive substances are investigated for possible internal contamination. The type of information provided on this internal monitoring varied between authorities, but all employers provided the calendar year in which an employee was monitored for the first time for potential internal contamination by any radionuclide. ${ }^{10}$ All workers for whom such a date existed are referred to in this study as internally monitored workers.

INVESTIGATION OF PRIMARY INFERTILITY

To ensure that most subjects had completed their families, or their attempts to start a family, the present analysis is restricted to workers aged 40 years or more at the time of the survey. Also, workers were excluded if either the estimated date of conception (date of the end of pregnancy minus duration of gestation plus 14 days) of their first pregnancy or their first attempt for a pregnancy if they had primary infertility (estimated as 6 months before they consulted a doctor) was before employment in the nuclear industry.

Primary infertility was examined here in two slightly different, but overlapping, ways. A worker was categorised as having primary infertility if they reported that they first consulted a doctor about their fertility at least 6 months after they were first employed by the nuclear industry and had either (a) never achieved a recognised pregnancy, or $(b)$ never achieved a viable pregnancy that lasted beyond 24 weeks of gestational age.

STATISTICAL METHODS

All analyses were performed with Stata statistical software. ${ }^{13}$ All $\mathrm{p}$ values quoted are two sided and values less than 0.05 were taken to indicate significance.

In all analyses the denominator consisted of all workers aged over 40 years at the time of the survey who had attempted to have their first child after joining the industry, whether or not they were successful.

The effect of parental exposure to radiation on risk of primary infertility was estimated by logistic regression, with the non-monitored group as baseline. Significance was assessed with the likelihood ratio test. Odds ratios (ORs) were adjusted for age at first consultation with a doctor about infertility (or age at first conception for the comparison group) and calendar period in 5 year bands. Trends in dose were examined among monitored workers, using the median dose to characterise all workers in a particular dose category. Cumulative dose before consultation with a doctor for the primary infertile group, or to conception of the first pregnancy for those in the comparison group, were cumulated from annual dose records with pro rata scaling of the annual doses in the year of consultation or conception.

\section{Results}

Overall, 5353 men and 603 women who were aged 40 years or more at the time of our survey joined the nuclear industry before starting, or attempting to start, their families (table 1). Of these, $2.6 \%$ men, and $3.7 \%$ women were categorised as having primary infertility (with no recognised pregnancies). When the definition was extended to include those who reported one or more fetal deaths occurring before 24 weeks of gestation, the proportions increased to $3.0 \%$ and $4.8 \%$ for men and women respectively. There is a clear trend for increasing prevalence of infertility with increasing age at first consultation with a doctor (table $1)$.

The median dose of radiation received before consultation with a doctor for infertility, or to conception, was $12.3 \mathrm{mSv}$ (5th to 95th percentiles: $0.2-515.2 \mathrm{mSv}$ ) for men and 5.9 $\mathrm{mSv}$ (5th to 95th percentiles: $0.1-57.1 \mathrm{mSv}$ ) for women.

Among men, there was no evidence of an association between primary infertility (no pregnancies) and monitoring for exposure to ionising radiation, either overall (OR 1.0, 95\% confidence interval ( $95 \% \mathrm{CI}) \quad 0.7$ to 1.4 , 
Table 1 Reported primary infertility * by age at first consultation for infertility or conception of first child (if no fertility problem)

\begin{tabular}{lccc}
\hline $\begin{array}{l}\text { Age at first consultation for } \\
\text { infertility or conception of } \\
\text { first child (if no fertility } \\
\text { problem) }\end{array}$ & All workerst & $\begin{array}{l}\text { Primary infertility, no } \\
\text { conceptions } \\
n(\%)\end{array}$ & $\begin{array}{l}\text { Primary infertility, no } \\
\text { pregnancies reaching } \geqslant 24 \\
\text { weeks } \\
n(\%)\end{array}$ \\
\hline $\begin{array}{l}\text { Male workers: } \\
<25\end{array}$ & 857 & $5(0.6)$ & $5(0.6)$ \\
$25-29$ & 2348 & $49(2.1)$ & $55(2.3)$ \\
$30-34$ & 1415 & $50(3.5)$ & $57(4.0)$ \\
$35-39$ & 550 & $23(4.2)$ & $27(4.9)$ \\
$\geqslant 40$ & 183 & $14(7.7)$ & $20(10.9)$ \\
Total & 5353 & $141(2.6)$ & $164(3.0)$ \\
& & $\chi^{2}{ }_{\text {trend }}: \mathrm{p}<0.0001$ & $\chi^{2}{ }_{\text {trend }}: \mathrm{p}=0.002$ \\
Female workers: & 175 & $0(0)$ & $2(1.1)$ \\
$<25$ & 245 & $10(4.1)$ & $6(5.9)$ \\
$25-29$ & 117 & $5(4.3)$ & $8(14.3)$ \\
$30-34$ & 56 & $7(12.5)$ & $1(10.0)$ \\
$35-39$ & 10 & $0(0)$ & $29(4.8)$ \\
$\geqslant 40$ & 603 & $22(3.7)$ & $\chi^{2}{ }_{\text {trend }}: \mathrm{p}=0.002$ \\
Total & & $\chi^{2}{ }_{\text {trend }}: \mathrm{p}<0.0001$ & \\
& & &
\end{tabular}

*Primary infertility defined as (a) having consulted a clinician about problems achieving a pregnancy, and never having achieved a pregnancy; and $(b)$ having consulted a clinician about problems achieving a pregnancy, and never having achieved a pregnancy lasting $\geqslant 24$ weeks of gestation.

†Aged $\geqslant 40$ at time of survey, and who had been employed when their first child was conceived, or when they were first attempting to have a child.

$\mathrm{p}=1.0$ ) or with increasing dose (trend among monitored; $p=0.34$, table 2 ). The analysis of primary infertility including fetal deaths before 24 weeks of gestation gave similar results. Investigation of the effect of monitoring and dose within 6 months of the first consultation for infertility (or estimated date of first conception in the comparison group) produced similar results (data not shown).
For women, although the risk of primary infertility was higher among those who were monitored than among those who were not (OR 1.6, 95\% CI 0.6 to $4.3, \mathrm{p}=0.33$ for primary infertility with no pregnancies; OR $2.1,95 \%$ CI 1.0 to $4.8, p=0.07$ for primary infertility with no pregnancies beyond 24 weeks) the numbers are small and there was no evidence of a trend with dose $(p=0.34$ and 0.77 for primary infertility with no pregnancies and with no pregnancies beyond 24 weeks, respectively). Analysis of monitoring and dose received within 6 months of the first consultation for infertility (or estimated date of first conception in comparison group) produced similar results (data not shown).

\section{Discussion}

The nuclear industry family study was set up to investigate the reproductive health of nuclear workers within the United Kingdom and the health of their offspring. An important component of reproductive health is the ability to conceive a pregnancy which results in the birth of a healthy child. Within the group of workers studied here-all of whom were aged 40 years or more at the time of the survey and started their families, or attempted to do so, after joining the industry- $2.6 \%$ of the men had never fathered a pregnancy and $3.0 \%$ had only fathered pregnancies which ended in miscarriage. All had consulted a doctor because of these difficulties.

Table 2 Reported primary infertility ${ }^{*}$ by monitoring statust before date of first consultation for infertility or conception of first child (if no fertility problem)

\begin{tabular}{|c|c|c|c|c|c|}
\hline \multirow{2}{*}{$\begin{array}{l}\text { Exposure before date of first consultation for infertility } \\
\text { or conception of first child (if no fertility problem) }\end{array}$} & \multirow{2}{*}{$\begin{array}{l}\text { All } \\
\text { workers } \neq\end{array}$} & \multicolumn{2}{|c|}{$\begin{array}{l}\text { Primary infertility, no } \\
\text { conceptions }\end{array}$} & \multicolumn{2}{|c|}{$\begin{array}{l}\text { Primary infertility, no } \\
\text { pregnancies reaching } \geqslant 24 \\
\text { weeks }\end{array}$} \\
\hline & & $n(\%)$ & ORS $(95 \% C I)$ & $n(\%)$ & $O R \int(95 \% C I)$ \\
\hline \multicolumn{6}{|l|}{ Male workers: } \\
\hline Not monitored & 1691 & $42(2.5)$ & 1.0 & $51(3.0)$ & 1.0 \\
\hline Monitored & 3662 & $99(2.7)$ & $1.0(0.7$ to 1.4$)$ & $113(3.1)$ & $0.9(0.7$ to 1.3$)$ \\
\hline Monitored, external only & 2364 & $68(2.9)$ & $1.1(0.7$ to 1.6$)$ & $75(3.2)$ & $1.0(0.7$ to 1.4$)$ \\
\hline Monitored, external and internal & 1298 & $31(2.4)$ & $0.8(0.5$ to 1.4$)$ & $38(2.9)$ & $0.9(0.6$ to 1.3$)$ \\
\hline \multicolumn{6}{|c|}{ Monitored, cumulative external dose $(\mathrm{mSv})^{\star \star}$} \\
\hline $0-2.49$ & 820 & $18(2.2)$ & $0.8(0.4$ to 1.4$)$ & $19(3.0)$ & $0.7(0.4$ to 1.2$)$ \\
\hline $2.5-9.99$ & 862 & $24(2.8)$ & $1.0(0.6$ to 1.7$)$ & $27(2.3)$ & $1.0(0.6$ to 1.6$)$ \\
\hline $10-19.99$ & 555 & $14(2.5)$ & $1.0(0.5$ to 1.8$)$ & $16(3.1)$ & $0.9(0.5$ to 1.7$)$ \\
\hline $20-49.99$ & 767 & $24(3.1)$ & $1.2(0.7$ to 2.0$)$ & $26(2.9)$ & $1.1(0.7$ to 1.8$)$ \\
\hline$\geqslant 50$ & 655 & $19(2.9)$ & $1.0(0.6$ to 1.8$)$ & $25(3.4)$ & $1.1(0.7$ to 1.8$)$ \\
\hline Monitored, cumulative dose $\geqslant 100 \mathrm{mSv}$ & 285 & $9(3.2)$ & $1.0(0.5$ to 2.2$)$ & $9(3.2)$ & $0.8(0.4$ to 1.7$)$ \\
\hline \multicolumn{6}{|l|}{ Female workers: } \\
\hline Not monitored & 457 & $15(3.3)$ & 1.0 & $18(3.9)$ & 1.0 \\
\hline Monitored & 146 & $7(4.8)$ & $1.6(0.6$ to 4.3$)$ & $11(7.5)$ & $2.1(1.0$ to 4.8$)$ \\
\hline Monitored, external only & 100 & $5(5.0)$ & $1.7(0.6$ to 5.0$)$ & $8(8.0)$ & $2.3(0.9$ to 5.7$)$ \\
\hline Monitored, external and internal & 46 & $2(4.4)$ & $1.5(0.3$ to 7.2$)$ & $3(6.5)$ & $1.8(0.5$ to 6.5$)$ \\
\hline \multicolumn{6}{|l|}{ Monitored, cumulative external dose (mSv) } \\
\hline $0-2.49$ & 40 & $4(10.0)$ & $3.7(1.0$ to 13.4$)$ & $5(12.5)$ & $3.9(1.3$ to 12.2$)$ \\
\hline $2.5-9.99$ & 59 & $2(3.4)$ & $1.1(0.2$ to 5.2$)$ & $3(5.1)$ & $1.3(0.4$ to 4.6$)$ \\
\hline $10-19.99$ & 19 & $0(0$ & 0.0 & $0(0)$ & 0.0 \\
\hline $20-49.99$ & 24 & $1(4.2)$ & $1.7(0.2$ to 14.3$)$ & $3(12.5)$ & $4.2(1.1$ to 16.4$)$ \\
\hline$\geqslant 50$ & 4 & $0(0)$ & 0.0 & $0(0)$ & 0.0 \\
\hline Monitored, cumulative dose $\geqslant 100 \mathrm{mSv}$ & 0 & $0(0)$ & 0.0 & $0(0)$ & 0.0 \\
\hline
\end{tabular}

* Primary infertility defined as (a) having consulted a clinician about problems achieving a pregnancy, and never having achieved a pregnancy; and (b) having consulted a clinician about problems achieving a pregnancy, and never having achieved a pregnancy lasting $\geqslant 24$ weeks of gestation.

†Presence or absence of monitoring for ionising radiation before first consultation for infertility (or estimated date of conception of first child for those with no reported fertility problem).

$\ddagger$ All workers aged $\geqslant 40$ at survey, who reported first attempting to have children after date of first employment. Date of first attempt at pregnancy taken as 6 months before first consultation for problems achieving pregnancy (or estimated date of conception of first child if no fertility problem reported).

ऽOdds ratio adjusted for age at first consultation or first conception if no fertility problems and year of first consultation or first conception if no fertility problems.

- Baseline group for all odds ratios.

${ }^{\star \star}$ For three men the dose before date of first conception was uncertain. No workers with fertility problems had uncertain doses before date of first consultation. 
A higher proportion of the women reported no pregnancies despite trying $(3.7 \%)$, or no pregnancies lasting 24 weeks (4.8\%).

The overall levels of primary infertility are similar to those reported elsewhere. A study of medical radiographers, which used a similar survey design, reported comparable figures: $3.0 \%$ of men and women reported primary infertility, defined as consultation with a doctor and no pregnancies. $^{8}$ Similarly, a study of women who had been treated with $x$ rays for adolescent idiopathic scoliosis reported that $3.8 \%$ had an unsuccessful attempt at pregnancy. ${ }^{4}$ Equivalent figures for the general population are not available, but a survey of women in one health authority of the United Kingdom found that $3.0 \%$ were involuntarily childless at the end of their reproductive lives. ${ }^{14}$

Among men, the prevalence of primary infertility was almost identical in monitored and non-monitored workers. Very few comparable studies have been conducted, reflecting the difficulty in obtaining accurate information on male infertility. Almost all the studies of radiation exposure and infertility in men relate to survivors of childhood cancer, the largest of these being a retrospective cohort study of over 2000 survivors of childhood and adolescent cancer in the United States. ${ }^{5}{ }^{6}$ This study used the number of years between marriage and first pregnancy as a measure of fertility and found that men who had been treated for cancer in childhood had 25\% lower fertility than their male siblings. However, investigation of the method of treatment showed that chemotherapeutic agents had a much stronger effect on time to first pregnancy than treatments involving $x$ rays.

For women, although there was some suggestion of an association between primary infertility and monitoring, this was not significant and there was no trend with dose. The small numbers and consequent low power limit the conclusions that can be drawn. In the context of other studies that have examined reproductive outcome in women exposed to ionising radiation, a recent study of over 2000 Canadian women who received $x$ ray treatment for adolescent idiopathic scoliosis reported a 30\% excess in unsuccessful attempts at pregnancy compared with randomly selected members of the general population. ${ }^{4}$ No evidence of a relation with dose was found and the authors concluded that the effect could have resulted from chance alone. ${ }^{15}$ Another Canadian study reported that women treated with abdominal pelvic radiation for cancer in childhood had reduced fertility, the effect increasing with dose received. ${ }^{7}$ However, the doses received by these patients were far higher than the doses received by women in this study. Previous studies on survivors of cancer noted longer intervals between marriage and first pregnancy ${ }^{56}$ or higher reported fertility problems. ${ }^{16}$ Studies of survivors of the atomic bombs in Japan have generally found little association between exposure to radiation and adverse reproductive outcomes including subfertility. ${ }^{3}$

We need to consider whether the results of this study could have resulted from limitations of the method. Our response rate was high, and there is little evidence of unusual patterns of reproductive outcome in the responders overall compared with other studies of occupation and reproduction. ${ }^{817}$ We cannot be sure that all adverse reproductive outcomes were reported, but it is unlikely that reproductive events occurring after employment in the nuclear industry would be deliberately omitted. Further, exposure data were obtained from employers rather than workers, and linkage to reproductive events was unbiased by recall of exposure to radiation. We thus consider it improbable that response or recall bias could account for the results found here. There were sufficient data from men to provide adequate power for the analyses. For the findings for women, we must consider the possible role of selection bias. It has been suggested that women who are childless tend to remain in the workforce, and thus inflate measures of prevalence of infertility. ${ }^{18}$ We attempted to avoid this bias by including past, as well as current, workers. Overall, we do not consider that the results reported here could be explained by limitations in the method.

In conclusion, this study found no evidence of an association between exposure of men to ionising radiation and primary infertility. For female workers there was some evidence of increased risk of infertility in monitored compared with non-monitored workers, but the numbers were too small, and hence the statistical power of the study too low, to enable a firm conclusion to be drawn. However, in the light of the finding of an increased risk of fetal loss in the pregnancies of female nuclear industry workers, ${ }^{12}$ the suggestion of an increased risk for women perhaps justifies further research on the reproductive health of women whose work involves exposure to ionising radiation.

We thank the many people who contributed to the study, including; management and workforce representatives who gave permission for the study to be conducted, industry staff who worked with us, and most importantly the study participants themselves. We also acknowledge the many staff who worked on the project, including Pat Ansell, Aurora Berra, Patricia Fraser, Elizabeth Hilton, Juliet Jain, Angela MacCarthy, Tuyet Ngyen, Margo Pelerin, Patrick Sampson, Moya Simmons, and Amanda Thomas. We our grateful to the Scientific Steering Group-Eva Alberman, Tim Bishop, Martin Bobrow, and Dudley Goodhead-for their support and guidance. The study was Executive. Contributors: PD (guarantor) participated in protoExecutive. Contributors: PD (guarantor) participated in proto-
col design, data collection, analysis, and writing the paper; NM col design, data collection, analysis, and writing the paper; NM
participated in protocol development, data collection, analysis, participated in protocol development, data collection, analysis,
and writing the paper; ER initiated the research and participated in protocol design, data collection, analysis, and writing the paper; GD participated in data collection and analysis; PS initiated the research and participated in protocol design and writing the paper; VB initiated the research and participated in protocol design. Competing interests: PS has received funding from British Nuclear Fuels for research on the health of Sellafield workers.

1 Committee on the Biological Effects of Ionising Radiations BEIR V). Health effects of exposure to low levels of ionising adiation. National Academy Press, Washington DC 1990. 2 Kline J, Stein Z, Susser M. Conception to birth. New York: Oxford University Press, 1989

3 Neel JV, Lewis SE. The comparative radiation genetics of humans and mice. Annu Rev Genet 1990;24:327-62.

4 Goldberg MS, Mayo NE, Levy AR, et al. Adverse reproductive outcomes among women exposed to low levels of ionising radiation from diagnostic radiography for adolescent idiopathic scoliosis. Epidemiology 1998;9:271-8.

5 Byrne J, Mulvihill J, Myers M, et al. Effects of treatment on Byrne J, Mulvihil J, Myers M, et al. Effects of treatment on
fertility in long-term survivors of childhood or adolescent fertility in long-term survivors of childhood

6 Byrne J. Long-term genetic and reproductive effects of ionising radiation and chemotheraputic agents on cancer patients and their offspring. Teratology 1999;59:210-15. 
7 Chiarelli AM, Marrett LD, Darlington G. Early menopause and infertility in females after treatment for chilhood cancer diagnosed 1964-88 in Ontario, Canada. Am $\mathcal{F}$ Epidemiol 1999:150:245-54.

8 Roman E, Doyle P, Ansell P, et al. Health of children born to medical radiographers. Occup Environ Med 1996;53:73-9.

9 Henderson J, Baker HWG, Hanna PJ. Occupation-related male infertility: a review. Clinical Reproduction and Fertility male infertility:

10 Maconochie N, Doyle P, Roman E, et al. Nuclear industry family study: methods and description of a United Kingdom study linking occupational information held by employers to reproduction and child health. Occup Environ Med 1999;56:793-801.

11 Roman E, Doyle P, Maconochie N, et al. Cancer in children of nuclear industry employees: report from the nuclear industry family study. BMF 1999;318:1443-50.
12 Doyle P, Maconochie N, Roman E, et al. Fetal death and congenital malformation in the pregnancies of nuclear Lancet 2000;356:1293 300 .

13 Statacorp. Stata statistical software; release 5.0. Texas, USA: Stata Corporation, 1996.

14 Gunnell DJ, Ewings P. Infertility prevalence, needs assessment and purchasing. F Public Health Med 1994;16:29-35.

5 Kline J. Does maternal exposure to radiation before conception affect reproduction? Epidemiology 1998;9:271-8.

16 Hawkins MM, Smith RA. Pregnancy outcomes in childhood cancer survivors: probable effects of abdominal radiation. Int $\mathcal{F}$ Cancer 1989;43:399-402.

17 Doyle P, Roman E, Beral V, et al. Spontaneous abortion in dry cleaning workers potentially exposed to perchloroethylene. Occup Environ Med 1997;54:848-53.

18 Joffe $M$. Biases in research on reproduction and women's work. Int f Epidemiol 1985;14:118-23.

\section{Vancouver style}

All manuscripts submitted to Occup Environ Med should conform to the uniform requirements for manuscripts submitted to biomedical journals (known as the Vancouver style.)

Occup Environ Med, together with many other international biomedical journals, has agreed to accept articles prepared in accordance with the Vancouver style. The style (described in full in the $\mathcal{F} A M A[1]$ ) is intended to standardise requirements for authors, and is the same as in this issue.

References should be numbered consecutively in the order in which they are first mentioned in the text by Arabic numerals on the line in square brackets on each occasion the reference is cited (Manson[1] confirmed other reports[2][3][4][5]). In future references to papers submitted to Occup Environ Med should include: the names of all authors if there are three or less or, if there are more, the first three followed by et al; the title of journal articles or book chapters; the titles of journals abbreviated according to the style of Index Medicus; and the first and final page numbers of the article or chapter. Titles not in Index Medicus should be given in full.

Examples of common forms of references are:

1 International Committee of Medical Journal Editors. Uniform requirements for manuscripts submitted to biomed journals. FAMA 1993;269:2282-6.

2 Soter NA, Wasserman SI, Austen KF. Cold urticaria: release into the circulation of histmaine and eosinophil chemotactic factor of anaphylaxis during cold challenge. N Engl F Med 1976;294:687-90.

3 Weinstein L, Swartz MN. Pathogenic properties of invadWeinstein L, Swartz MN. Pathogenic properties of invad-
ing micro-organisms. In: Sodeman WA Jr, Sodeman WA, eds. Pathologic physiology, mechanisms of disease. Philadelphia: W B Saunders, 1974:457-72.
. 